\title{
Optimization of the ITER EC H\&CD Functional Capabilities while Relaxing the Engineering Constraints
}

\author{
D. Farina ${ }^{*}$, M. Henderson ${ }^{\dagger}$, L. Figini*, G. Saibene ${ }^{* *}$, T. Goodman ${ }^{\ddagger}$ K. Kajiwara ${ }^{\S}$, T. \\ Omori $^{\dagger}$, E. Poli ${ }^{i l}$, D. Strauss" and K.Takahashi ${ }^{\dagger \dagger}$ \\ *Istituto di Fisica del Plasma, CNR, EURATOM-ENEA-CNR Association, Milano, Italy \\ ${ }^{\dagger}$ ITER Organization, Saint Paul Lez Durance, France \\ ${ }^{* *}$ Fusion for Energy, Barcelona, Spain \\ ${ }^{\ddagger}$ Centre de Recherches en Physique des Plasmas, CRPPĐEPFL, Lausanne, Switzerland \\ $\S_{J A E A, ~ N a k a, ~ J a p a n}$ \\ "Max-Planck-IPP, Euratom Association, Garching, Germany \\ "Karlsruhe Institute of Technology, Assoc. KIT-EURATOM, Karlsruhe, Germany \\ ${ }_{\dagger}^{\dagger} J A E$ A, Naka, Japan
}

\begin{abstract}
The work on optimization of the ECH\&CD system in ITER is presented with focus on its functional capabilities. Since the conceptual design of the system it has evolved both in goals and functionalities, by considering an expanded range of H\&CD applications. A large effort has been devoted to a better integration of the two types of launcher, the equatorial and the upper, both from the point of view of the performance and the impact on the engineering constraints of the design.
\end{abstract}

Keywords: Heating and current drive, electron cyclotron waves, MHD stabilization

PACS: $52.35 . \mathrm{Hr}, 52.50 . \mathrm{Sw}, 52.55 . \mathrm{Wq}$

\section{INTRODUCTION}

A 24MW CW Electron Cyclotron Heating and Current Drive (EC H\&CD) system operating at $170 \mathrm{GHz}$ is to be installed for the ITER tokamak. The power will be injected via two launcher types (one equatorial, EL, and four upper, UL, launchers) to assist and sustain ITER operations in various scenarios since the early phase of ITER. The EC system was designed initially with the goal to fulfill some specific high priority scientific objectives: central heating, bulk current drive and MHD control. The EC H\&CD functionalities were partitioned between the two launchers based on the accessibility and ECCD deposition profiles. The UL will provide narrow peaked current density profiles over the outer half of the plasma suitable for MHD control (NTMs and sawtooth mainly), while the EL will drive larger current with by wider profiles, suitable for central heating and bulk current drive. On the basis of the desired functionalities and of the engineering constraints the design up to 2011 consisted in: a) the Equatorial launcher equipped with 8 beams per three rows (Top, Mid and Low), injected at fixed poloidal angle and toroidally steered in the range from $20^{\circ}$ to $\approx 42^{\circ}$; b) the Upper Launcher with 4 beams per two mirrors (Upper and Lower Steering Mirrors), at fixed toroidal angle around $20^{\circ}$ and poloidally steerable by $24^{\circ}$.

The proposed partitioning is able to satisfy the main required functionalities, however some weaknesses of the system were identified, the main one being limited EC access at mid radius. Note that prior to 2009, the EL toroidal steering had particular access restrictions, which included limited access toward the center from the top and lower steering rows, limited access at mid radius as the beam is steered far from the resonance resulting in less than $100 \%$ first pass absorption, less than efficient driven current at mid radius due to the above and to non-optimal toroidal and poloidal injection angles. Some of these limitations were mitigated by limiting the large toroidal steering angles to avoid beam shine through. For the investigated scenarios, the EL can not access beyond $\rho \geq 0.45$ (being $\rho$ the normalized toroidal radius, i.e., the square root of the toroidal flux normalized to the edge value), which significantly limits the role that the EL can play in current profile tailoring in advanced and hybrid scenarios. Recall that ECCD is the prime tool in ITER for depositing current drive locally for controlling the plasma current profile. For this reason, a modification of the UL was proposed in order to have one of the two mirrors (USM) aiming at the inner plasma region via larger poloidal and toroidal angles. However, as the Upper Launcher is designed for localized and peak current density profile over the outer half of the plasma cross section, its capability of driving current at mid-radius is not optimal. The EC driven current localization and amount is shown in Figure 1 for injection from EL (toroidal steering 

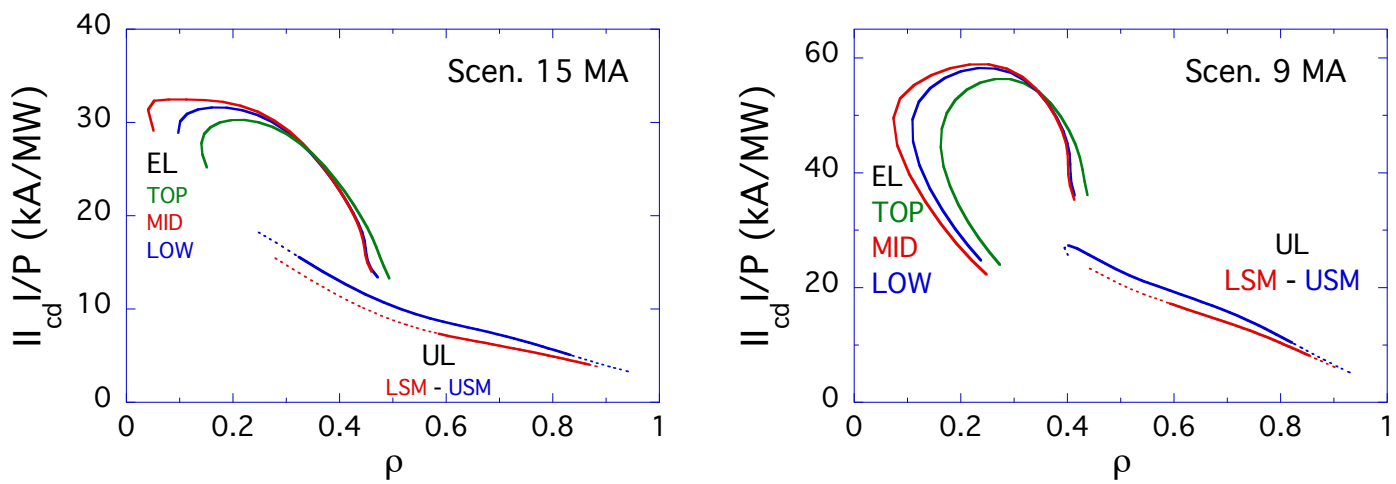

FIGURE 1. EC driven current versus radius for EL injection from the three rows and toroidal steering at fixed poloidal angle and for UL injections from the upper and lower mirrors for poloidal steering at fixed toroidal angle in the reference ITER inductive scenario at $15 \mathrm{MA}$ (left) and the advanced scenario at $9 \mathrm{MA}$ (right). The EL results are obtained using the foreseen range of toroidal angles $20^{\circ} \leq \beta \leq 42^{\circ}$, and the UL results for an extended poloidal range $\left(\Delta \alpha=40^{\circ}\right)$. The solid curve for the UL corresponds to the actual poloidal steering interval $\left(\Delta \alpha=24^{\circ}\right)$ and the range proposed in 2008-09.

case) and UL (poloidal steering case) for two reference ITER scenarios at burn, the inductive reference ITER scenario at $15 \mathrm{MA}$ and the advanced scenario at $9 \mathrm{MA}$. From the figure it is apparent that only a limited amount of power $(\approx 6.7$ MW) can be delivered to the region around mid radius, provided that the USM is designed to aim downwards.

At the same time, it was clear that the EC system could be improved in order to fill in the identified gaps and to extend its capabilities to an expanded range of challenging H\&CD applications in ITER, based also on the large experience developed on previous and present devices. The additional capabilities include break-down and rampup assist, disruption mitigation, co and counter combinations for current profile tailoring, and impurity control. The UL and EL steering range were modified prior to 2009, which provided access to achieve nearly all of these requirements. Since that date, additional capabilities have been identified, for example the use of the EC system for central heating applications in the intermediate toroidal field strengths between fundamental and second harmonic has been considered. This is an essential capability since it is critical for the initial $\mathrm{L}$ to $\mathrm{H}$-mode power scaling, which will determine if more $\mathrm{H} \& \mathrm{CD}$ power is required prior to DT operation.

In order to comply with the updated physics requirements, significant modifications to the Equatorial Launcher are proposed, which aim primarily at more than doubling the ECCD in the range of $0.45<\rho<0.6$. This can be achieved by changing the steering plane from toroidal to poloidal. The change will ensure maintaining a wider flexibility in applying EC power from the breakdown through to the beginning of the ramp up phase.

The proposed changes of EL allow for a better share of the functionalities between the two types of launchers, in particular, accessibility to inner plasma regions from the UL can be relaxed. In addition the changes aim at also relaxing the engineering design constraints on the two launchers, which include increasing the nuclear shielding potential, relaxing the rotation cycles of the steering mirrors (to avoid onset of cyclic fatigue) and introducing redundancy in the event of failure of a given steering mirror.

A poloidal steering EL was never integrated into the design as the time it took to re-design the BSM would risk the delivery of the EL for the first plasma. However at present the EL BSM requires a redesign arising from the request to remove the Be coating from the BSM surface. Removal of the Be simplifies the EL design and offers a reduction in cost for both the development and manufacturing phase. In light of this resource savings, a task for optimizing the EL driven current capabilities has been launched with the goal to revise the design of the launching angles.

Here we present a survey of recent investigations about the ECRH\&CD capabilities of the ITER system. Calculations have been performed using the beam-tracing code GRAY [1], with momentum conservation model for ECCD [2]. The ECCD capabilities are investigated injecting EC beams polarized as ordinary mode, varying the launching steering angles in a wide range. The poloidal and toroidal launching angles $(\alpha, \beta)$ are defined in terms of the cylindrical components of the wavevector, i.e., $\tan \alpha=k_{z 0} / k_{R 0}$ and $\sin \beta=k_{\phi}(\alpha>0$ means downwards injection and viceversa). Both single ray and beam calculations have been performed, the former being faster and accurate enough for the estimate of the total amount of current and its localization, the latter slower while more accurate whenever details on the current density profile are required.

In the course of the work, various ITER scenarios have been taken into consideration, here we refer to two classes of scenarios at burn. The first one is an ELMy inductive H-mode $15 \mathrm{MA}$ at full field $(5.3 \mathrm{~T})$ and its variants: the so-called 

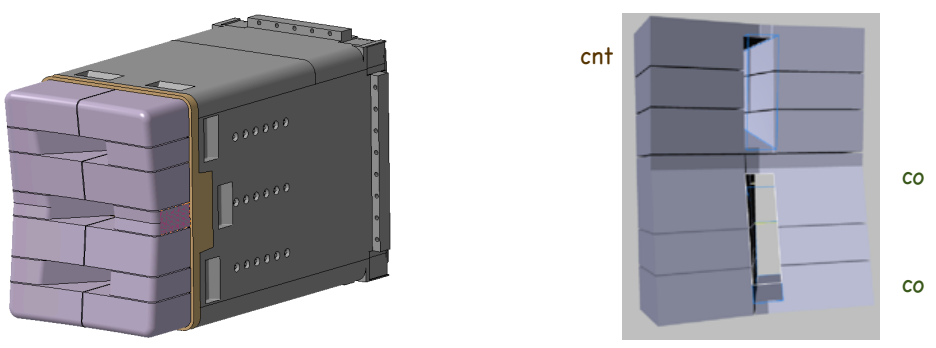

FIGURE 2. Sketch of the Equatorial Launcher for toroidal (left) and poloidal (right) steering.

Gribov scenario [3], Scenario 2 [4, 5], and more recent versions [6]. The second class correspond to a non-inductive scenario (9 MA) at full field and its variants. The obtained results are qualitatively similar and differ slightly depending on the variants, not affecting however the overall conclusions. In addition, quite recently simulations on a full time history of a reference inductive H-mode scenario have been performed for the UL [7, 8].

\section{EQUATORIAL LAUNCHER FUNCTIONALITIES}

The EL design is being developed by the JAEA team in collaboration with ITER [9], and enhancements of the design aimed at improved reliability and functionalities have been considered [10]. The main changes to date were driven by the physics access requirements, which introduced the poloidal tilt of the Top and Bottom mirrors and the flip of one row to provide counter $\mathrm{CD}$. Two different simulations conditions were considered with counter-injection either from Mid row or from Top row, without significant differences in the performances. This lead to the present EL design as illustrated in Fig.2. As mentioned above, further modifications to the EL are envisioned incorporating poloidal steering. The present configuration assumes that the mid and bottom mirrors drive co-current with the beams passing through the same BSM opening, thus minimizing the cut-out volume of the BSM and improving neutron shielding potential (see Fig.2).

Calculations of the EC driven current and access (shown in Fig. 1) for the inductive (15 MA) and the non-inductive scenario (9 MA) demonstrate that a quite large current is driven in the $9 \mathrm{MA}$ scenario due to the larger $T_{e} / n_{e}$ ratio (by a factor 2), i.e., to the larger CD efficiency [6]. The different EC localization in the two scenarios can be explained as due both to the larger temperature and to the outward shift of the plasma axis for the 9 MA Scenario. In addition second harmonic parasitic absorption is found to affect the ECCD performance in the high temperature 9MA scenario. For both scenarios, the EL could not access beyond $\rho \leq 0.45$, which significantly limits the role that the EL can be used in current profile tailoring in advanced and hybrid scenarios.

A study on EL optimization was performed aiming at avoiding the above deficiency. This study assessed the optimum injection angles from all $3 \mathrm{EL}$ steering mirrors, to maximize the driven current across the plasma cross section, concentrating on achieving EL access out to at least $\rho=0.6$. The study first scanned the EL over a wide range of launching angles for each EL row. These results are summarized in Fig. 3 where the contours of the absolute value of the EC driven current $I_{c d}$ and of the average radial position $\rho$ are shown in the $(\alpha, \beta)$ plane. The plots clearly illustrate that localized ECCD can be achieved beyond mid radius via a proper choice of the poloidal and toroidal launching angles.

Besides its primary functions, the EL will be used for plasma breakdown and burn through. The burn through application is of particular concern as the first pass absorption is extremely small. This can be enhanced by injecting O-mode at a shallow toroidal injection angle and then reflecting off the central column for High Field Side (HFS) Xmode injection. The HFS X-mode injection has a high first pass absorption for low $T_{e}-n_{e}$ plasmas. The polarization shift from $\mathrm{O}$ to $\mathrm{X}$-mode is achieved upon reflecting on the central column. Figure 4 illustrates the accessing range of the EL top mirror and UL lower mirror upon reflecting of the central column for the $(\alpha, \beta)$ steering range. Color contours indicate the $z$ value at which the ray trajectory intersects the cold EC resonance (located at $R=5.4 \mathrm{~m}$ ) at second pass into the plasma, i.e., after reflection from inner wall. In case of the EL, toroidal angles of $25^{\circ}$ have the beam center grazing the central column. For large $\beta$ values wall reflection does not occur because of a pure geometrical effect, so that polarization scrambling ( $\mathrm{O}$ to $\mathrm{X}$ ) via inner wall reflection cannot take place. In order to ensure a full beam reflection off the central column, a toroidal injection angle of $20^{\circ}$ is proposed for the top steering mirror. 

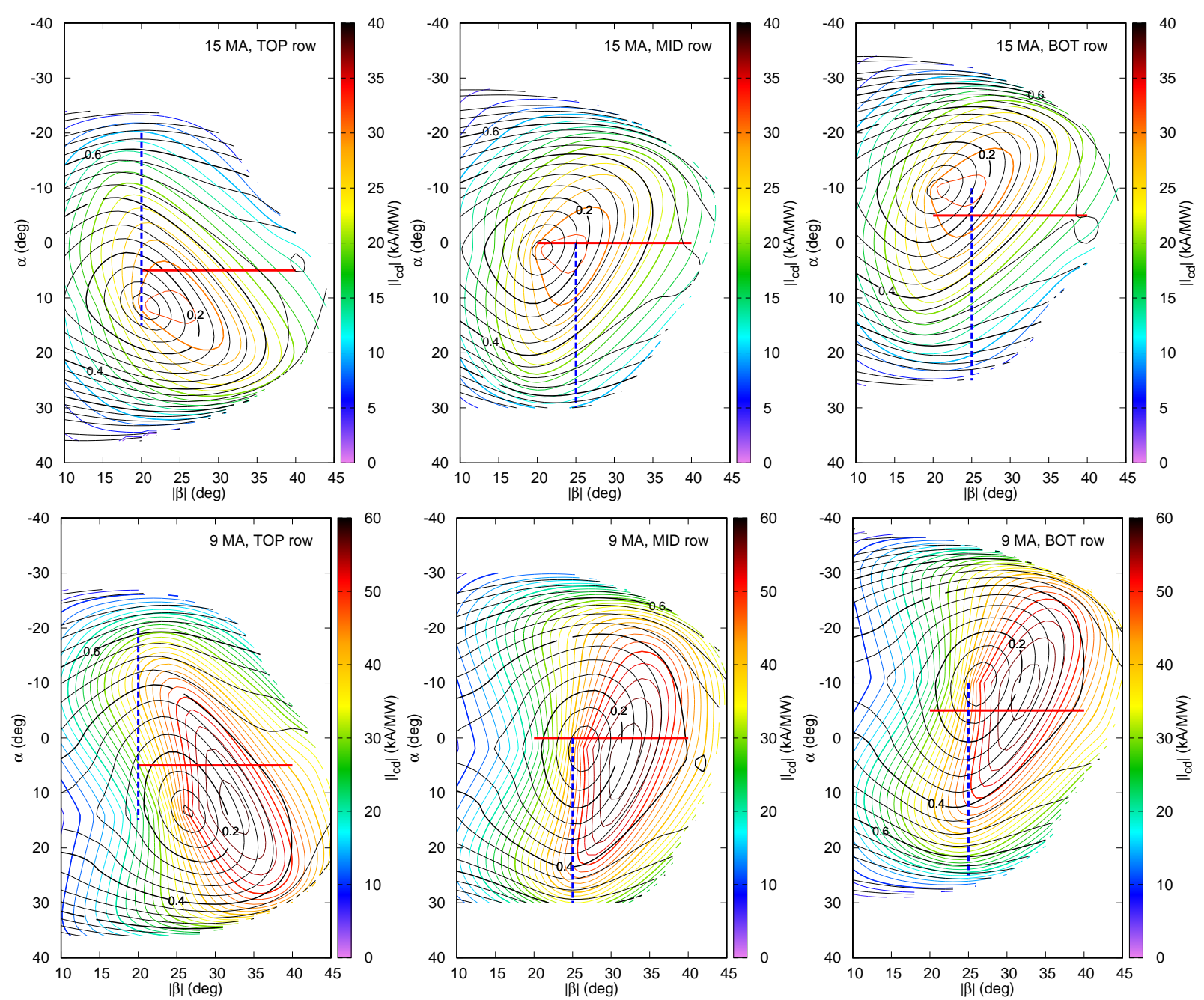

FIGURE 3. Contours of EC driven current $I_{c d}$ (colors) and radius of localization $\rho$ (black) in the space of launching angles $(\alpha, \beta)$, for the three steering mirrors for the $15 \mathrm{MA}$ (top row) and 9 MA (bottom row) scenario. The solid red line is the toroidal sweep path while the dashed blue vertical line the proposed poloidal sweep path.

In general we can say that poloidal steering plane offers a wider range of accessibility over the toroidal steering. Thus, having in mind the specific goals of each EL mirror a choice can be made for the "optimal" toroidal angle for poloidal steering (not the same at each row). Note that the poloidal steering offers an engineering advantage, in that the openings in the Blanket Shield Module (BSM) is reduced, increasing the neutron shielding potential of the launcher.

The EL top mirror is configured to drive counter ECCD, with primary objectives to drive counter current inside $\rho=0.4$, and to be used with co-ECCD to decouple heating and current drive in region $\rho<0.2$. The choice $\beta=20^{\circ}$ ensures the above functionality and offers a shallow toroidal angle to reflect off of the central column, as required by plasma break down and burn through functions allocated to the top steering mirror. This allows also the co-ECCD steering mirrors to have a larger toroidal angle to drive more current. Both the Mid and the Bottom mirrors are in co-ECCD with a toroidal injection angle of $25^{\circ}$, with primary objectives to drive as much current as possible in the range of $0.4<\rho<0.6$ and to be used with counter-ECCD for $\rho=0.2$.

A larger toroidal angle increases the driven current at mid radius for the 9 MA scenario, but then fails to achieve access $\rho<0.2$ for the $15 \mathrm{MA}$ scenario. The choice $\beta=25^{\circ}$ for the toroidal injection angle offers the best compromise between driving more current at mid-radius, while accessing inner regions for the $15 \mathrm{MA}$ scenario. Note that keeping the same toroidal injection angle for the middle and bottom rows allows the two beams to pass through the same BSM opening, minimizing the cut-out volume, which is optimum for neutron shielding as discussed above. 

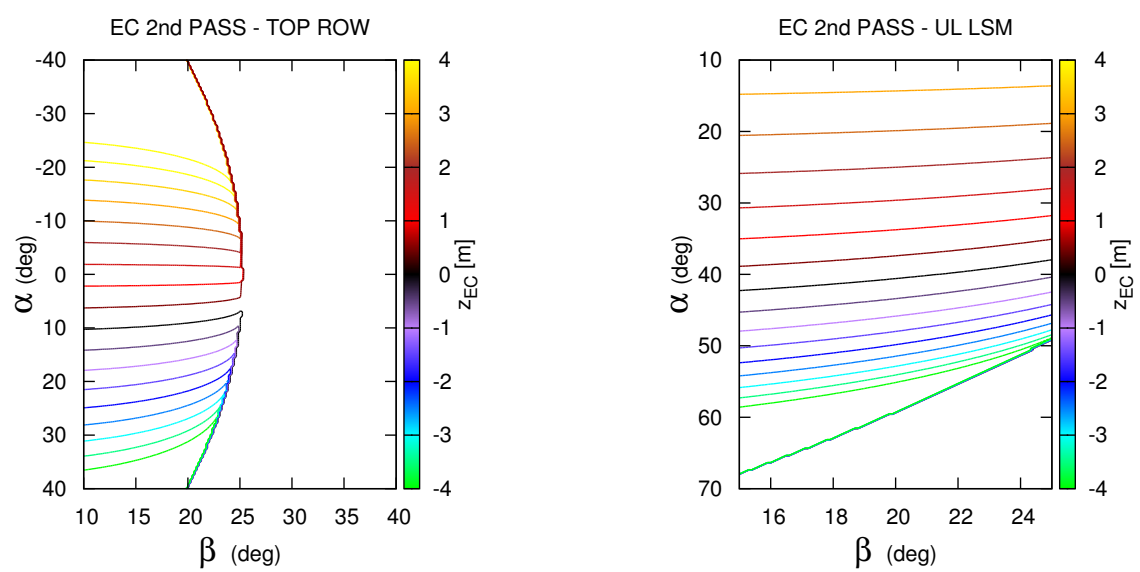

FIGURE 4. The steering range along the resonant layer for the EL top row (left) and from the UL lower steering mirror (right) upon reflection from the central column. Color contours indicate the $z$ value at which the ray trajectory intersects the EC resonance after reflection from inner wall.
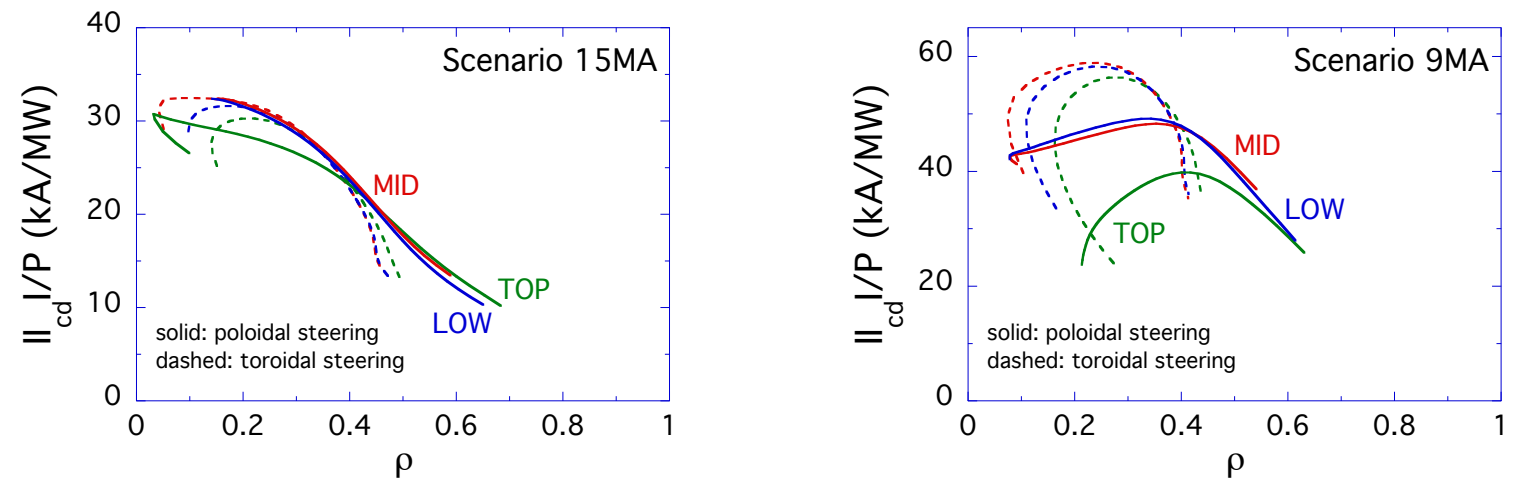

FIGURE 5. Driven current for pure poloidal (solid lines) and toroidal (dotted lines) steering in the two scenarios.

Numerical simulations have been performed with the GRAY code for an EC astigmatic beam with the following parameters: $w_{0 x}=12 \mathrm{~mm}, w_{0 y}=23.2 \mathrm{~mm}, d_{0 x}=505 \mathrm{~mm}, d_{0 y}=618 \mathrm{~mm}$, where $w_{0}$ is the beam waist and $d_{0}$ the waist distance from the launching point ( $x$ direction is in the horizontal plane, $y$ is perpendicular to $x$ and to the launched wave vector). Such a beam is a good approximation of the beams in the 2011 design of the EL and can provide a reasonable estimate of the ECRH\&CD profiles. The launching angles are as follows: i) Top row in counter-injection at $\beta=-20^{\circ}$ and $-20^{\circ} \leq \alpha \leq 15^{\circ}$; ii) Mid row in co-injection at $\beta=25^{\circ}$ and $0^{\circ} \leq \alpha \leq 35^{\circ}$; iii) Bottom row in co-injection at $\beta=25^{\circ}$ and $-10^{\circ} \leq \alpha \leq 25^{\circ}$. The comparison between the driven current via toroidal and poloidal steering is shown in Figure 5 for both scenarios and the three mirrors and the above ranges of the injection angles. It is clearly shown that poloidal sweep allows to extend the ECCD radial region up to $\rho \approx 0.6$ in both cases (except in cases of Mid row in the 9 MA case where a slightly lower value is found). This result is obtained at the cost that lower $I_{c d}$ is driven in the region $\rho<0.4$, especially in the $9 \mathrm{MA}$ case and from the top row. This is related to the fact that in case of toroidal steering the toroidal injection angle is varied in the range $20^{\circ}-40^{\circ}$, while in the case of poloidal steering the $\beta$ values are in the lower range ( $-20^{\circ}$ from Top row and $25^{\circ}$ from Mid and Bottom rows). Note however that, although reduced, the ECCD efficiency is still quite high.

\section{UPPER LAUNCHER FUNCTIONALITIES}

The UL design is being carried on under the F4E grant GRT-161 by ECHUL-CA, a Consortium of several European laboratories (KIT, CRPP, DIFFER-FOM, IPP and IFP-CNR) in collaboration with F4E and ITER. The UL is illustrated in Fig. 6. 
The Upper Launcher will be used for the control of MHD instabilities, primarily the neoclassical tearing mode (NTM) and then the sawtooth oscillation, which require that a current be generated within a narrow channel either inside the magnetic island or just outside, depending on the instability to be controlled [11]. On the basis of the ECCD analysis performed in recent years (see e.g. $[4,5]$ ), the toroidal injection angles were chosen in order to fulfill the requirement of NTM control on the $q=2$ and $q=3 / 2$ surfaces. In the preliminary design of the launcher the following values were found as the "optimal" ones for NTM control: $\beta=18^{\circ}$ for the LSM and $20^{\circ}$ for the USM. The $\mathrm{EC}$ driven current versus radius is shown in Fig. 1 for the two scenarios and the chosen $\beta$ values. Note that optimization was performed on the $15 \mathrm{MA}$ scenario, that is the one for which NTM control is highly desirable.

For a better sharing of the launchers capabilities, similarly to the analysis shown in the previous section for the EL an extensive ECCD investigation has been performed recently for the UL and both scenarios for launching angles in the range $25^{\circ} \leq \alpha \leq 70^{\circ}$ and $15^{\circ} \leq \beta \leq 25^{\circ}$. The beam parameters are those fixed in the preliminary design, i.e., focussed circular beams with $w_{0}=2.9 \mathrm{~cm}$ and $d_{0}=2.13 \mathrm{~m}$ for the USM and $w_{0}=2.1 \mathrm{~cm}$ and $d_{0}=1.62 \mathrm{~m}$ for the LSM. The results are summarized in Figs. 7, and 8 for injection from both mirrors and the two scenarios.

The UL performance has been analyzed in recent years making use of two criteria of NTM stabilization. The first one, based on the driven current density inside island [12], requires that the peak current density $J_{c d}$ exceeds the bootstrap current $J_{b s}$ by a factor of 1.2, i.e., $\eta_{N T M} \equiv J_{c d} / J_{b s}>1.2$, while the second one is based on the total current [13] and requires that $\eta_{N T M} w_{c d}>5 \mathrm{~cm}$ with $w_{c d}<5 \mathrm{~cm}$, being $w_{c d}$ the full width of the current profile measured in $\mathrm{cm}$. In support of the final UL design, the derivation of these criteria has been reviewed and their applicability range discussed [8]. It is found that the first criterion is valid for large deposition profiles while the second criterion for smaller deposition widths. For $w_{c d} \simeq 4.2 \mathrm{~cm}$ the two criteria lead to the same value of the power required for stabilization (that is also the minimum with respect to $\beta$ on the resonant surface), while at large width the required power is dominated by the first criterion and viceversa. Note that the UL "optimal" toroidal injection angles reported above were determined using the first criterion $\eta_{N T M}>1.2$.

The driven current density $J_{c d}$ and total driven current $I_{c d}$ and the ECCD localization are shown versus the injection angles for the reference ITER scenario at $15 \mathrm{MA}$ in Fig. 7, together with the $w_{c d}=5 \mathrm{~cm}$ contour that splits the space of launching angles in two regions. The upper left region is the one relevant for NTM stabilization since it corresponds to profile width smaller than $5 \mathrm{~cm}$. As it is clear from the plots, maximizing $J_{c d}$ (first criterion) or $I_{c d}$ (second criterion) yields a different value of the optimal toroidal angle. Whenever the beam width is kept small enough, the criterion on the total current prevails in most cases. The minimum power required for stabilization occurs at $\beta \approx 20^{\circ}$ in case of the USM (a bit lower on the $q=3 / 2$ and higher on the $q=2$ ), thus confirming the previous estimate, while for the LSM a $\beta$ values larger than $18^{\circ}$ should be preferred (see also [14]). Note that the values of peak current density and of its width depend on the beam characteristics, while, for the case of the UL, the total EC current and its localization are almost independent of the beam parameters. This explains the difference between the results from the two mirrors. For the final optical design, the effects leading to a broadening of the profile width, such that those due to diffusion, misalignment, beam overlapping, have to be taken into account in the choice of the optimal angle. Increasing $\beta$ may be beneficial because of the increase of $I_{c d}$, but only if $w_{c d}$ is kept below the optimum value.

In support of the final UL design investigations of the ECCD capabilities for NTM stabilization on the reference ITER scenario and its variants are being carried on focussing on the whole time history of the ITER discharge from ramp-up to ramp down and not only on the end of burn stage [8].

Additional functional capabilities of the UL have been considered such as sawtooth control and current profile tailoring that is the main EC goal in case of the advanced scenario. Improvements performed during the preliminary

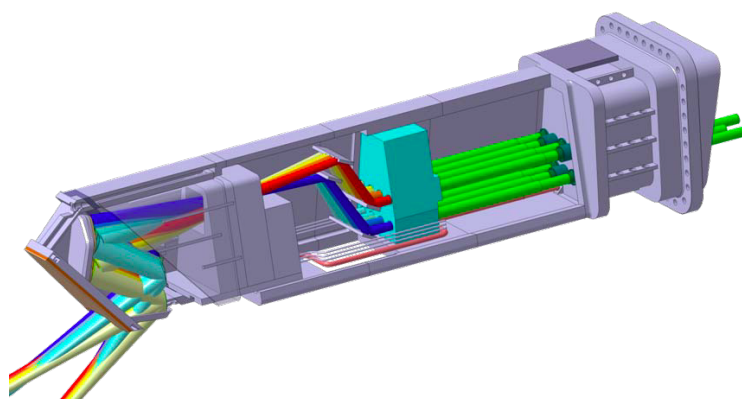

FIGURE 6. Sketch of the Upper Launcher. 

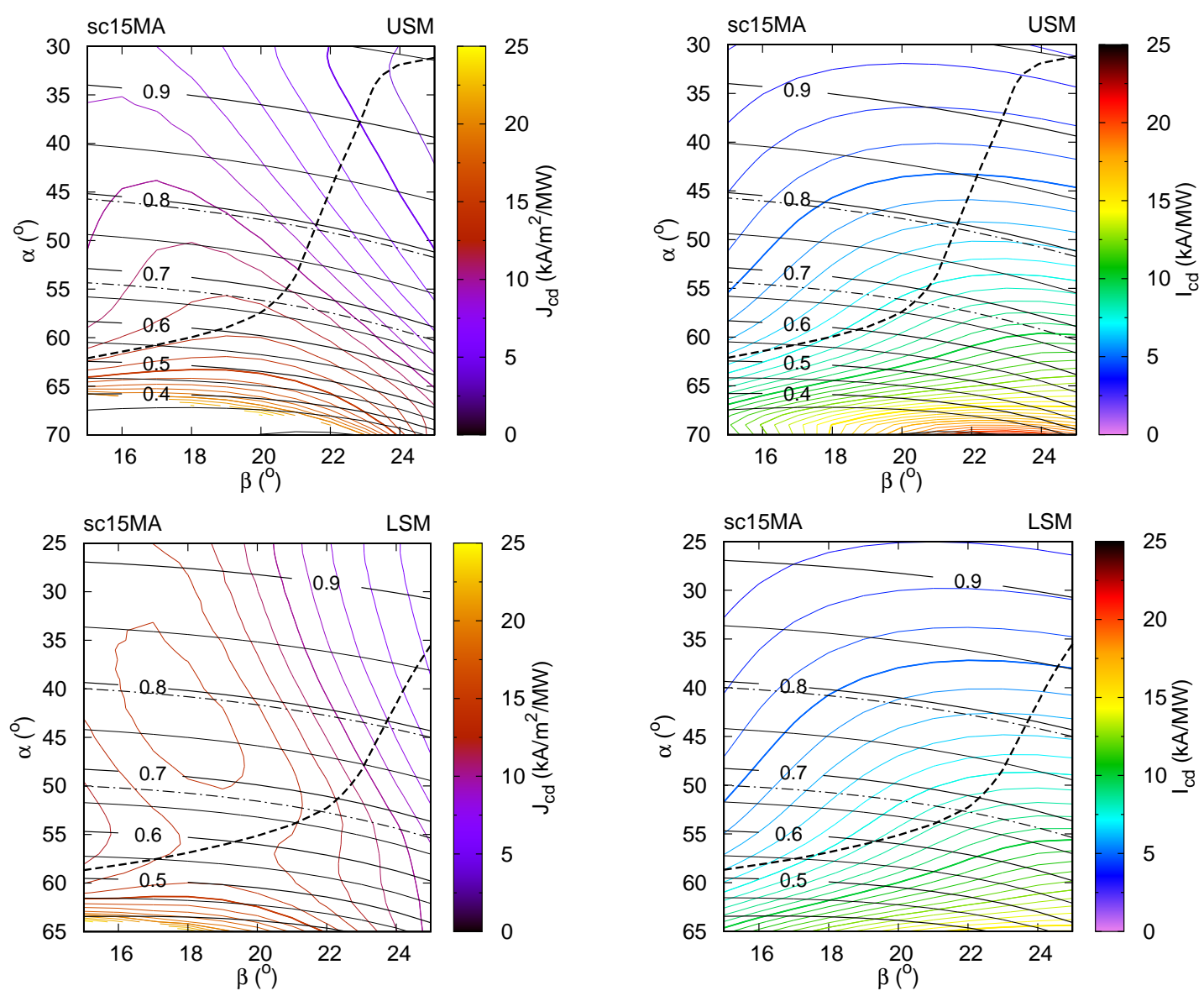

FIGURE 7. Top row: ECCD contours (color) and radius of localization $\rho$ (black) in the space of launching angles $(\alpha, \beta)$ for USM, with the EC driven current density $J_{c d}$ on the left plot and the total driven current $I_{c d}$ on the right plot, for the inductive scenario at 15 MA. Bottom row: same as in the top row for the LSM. The dash dotted contour $\rho \approx 0.79$ and 0.67 are the radius of the $q=2$ and $3 / 2$ surfaces respectively. The black dotted line corresponding to full width of the current profile $w_{c d}=5 \mathrm{~cm}$ splits the plot in two regions, with narrower and wider profile on the left and right side, respectively.

UL optical design phase allowed to increase the scanning range of the USM for the inductive scenario in order to access $0.3 \leq \rho \leq 0.5$, i.e., the $q=1$ surface, providing control of the sawtooth oscillation, while maintaining equivalent NTM stabilization efficiencies [5]. This can be accomplished by increasing the toroidal injection angle to improve the ECCD efficiency when aiming towards inner regions. The total driven current $I_{c d}$ and its localization are shown in Fig. 8 versus the injection angles for the advanced scenario at 9 MA. Quite a large current can be driven in a wide region, although the region $\rho \leq 0.4$ can not be accessed mainly because of geometrical reasons (the plasma center is shifted outward, see Fig.4 in [6]).

Another requested UL functionality is to provide EC assisted breakdown and burn through in support to the EL. A preliminary analysis shows that this is achievable via power injection from the LSM as it is shown in Fig.4. Further detailed investigation is in progress.

If the EL would move from toroidal to poloidal sweep, the requirement on the UL to access to inner plasma region would be relaxed. This fact would be beneficial from the point of the view of the engineering design since it would be easier to accommodate the beams in the Upper Launcher and reduce the BSM opening (see Fig.6).

\section{CONCLUSIONS}

The EC functional capabilities have been improved in a two step process. The first step was completed in 2009, which ensured EC power deposition across nearly the entire plasma cross section. This was achieved by separating out the 

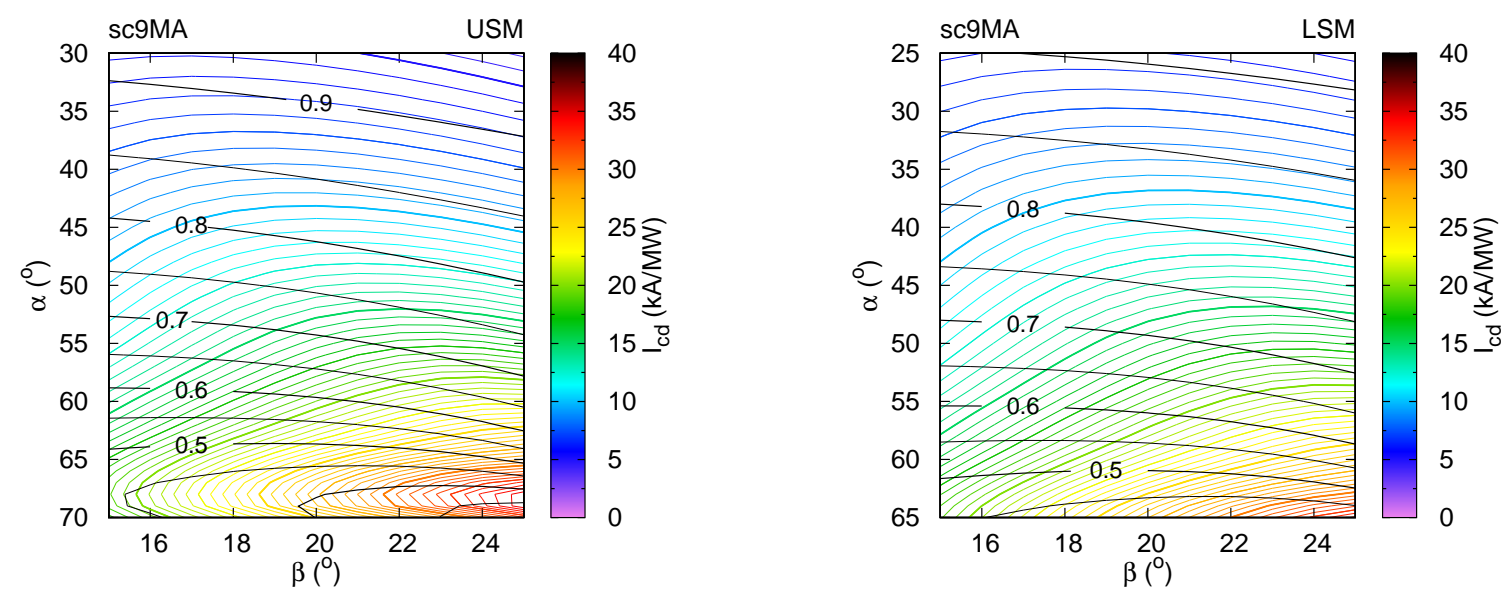

FIGURE 8. Contours of EC driven current $I_{c d}$ (color) and radius of localization $\rho$ (black) in the space of launching angles $(\alpha, \beta)$ for USM (left) and LSM (right) for the advanced scenario at 9 MA.

access range of the two UL steering mirrors. The upper steering mirror provided access into $\rho \geq 0.3$, filling in the large gap due to the limited access of the EL of $\rho \leq 0.45$. In addition the EL top and bottom mirrors were tilted providing a wider range of access, in particular additional central access.

The second step is now in progress, which introduces a poloidal steering EL. This change is introduced relatively late in the project development arising from a requirement to modify the EL BSM design (removing of the Be from the plasma facing surface). Analysis has demonstrated that a poloidal steering launcher can ensure access of the EL beams out to $\rho \leq 0.6$ and nearly double the EC driven current in the range of $0.45 \leq \rho \leq 0.6$. The additional driven current will be critical in control of the Hybrid scenarios and control of the Advanced scenario current density profile as well as extending the pulse length. This change also allows relaxation of the UL steering range, reducing the potential risk of cyclic fatigue of the UL steering mirror assemblies. In addition, the poloidal steering reduces the opening in the EL $\mathrm{BSM}$, improving the shielding potential and reducing the activation rates at the launcher back end.

The complete set of changes to the EC Launchers results in an EC system with increased H\&CD capabilities optimized for the three plasma scenarios (ELMy H-mode, Hybrid and Advanced) envisioned for ITER.

\section{ACKNOWLEDGMENTS}

This work was performed within a ITER Contract and a Fusion for Energy Grant GRT-161. The views expressed in this publication are the sole responsibility of the author and do not necessarily reflect those of ITER and Fusion for Energy.

\section{REFERENCES}

1. D. Farina, Fusion Sci. Technol., 52, 154 (2007)

2. N. B. Marushchenko et al, Fusion Sci. Technol., 55, 180 (2009)

3. R. Prater et al, Nucl. Fusion, 48, 035006 (2008)

4. G. Ramponi et al, Fusion Sci. Technol., 52, 193 (2007)

5. G. Ramponi et al, Nucl. Fusion, 48, 054012 (2008)

6. D. Farina et al, Nucl. Fusion, 52, 033005 (2012)

7. F. Koechl et al, Proc. 39th EPS Conf. \& 16th Int. Congress on Plasma Physics (Stockholm, Sweden), P1.042 (2012)

8. E. Poli et al, Proc. 40th EPS Conf. (Helsinki, Finland), Paper XXX (2013)

9. K. Takahashi et al, Nucl. Fusion, 48054014 (2008)

10. K. Takahashi et al, Proc 24th Fusion Energy Conference IAEA (San Diego, USA), Paper ITR/P1-05 (2012)

11. M. A. Henderson et al, Nucl. Fusion, 48054013 (2008)

12. H. Zohm et al., Plasma Physics and Controlled Fusion, 49 B341 (2007)

13. O. Sauter et al., Plasma Physics and Controlled Fusion, 52025002 (2010)

14. N. Bertelli et al., Nucl. Fusion, 51103007 (2011) 